\title{
Metagenomic Approach in Detection of Pathogenic Viruses
}

Nipuni TKR and Dassanayake RS ${ }^{*}$

*Corresponding author: Dassanayake RS, Department of Chemistry, University of Colombo, Colombo 03, Sri Lanka, Tel: 94-11-2503 367; Email: rsdassanayke@chem.cmb.ac.lk

Rec date: May 08, 2015; Acc Date: June 04, 2015; Pub date: June 06, 2015

Copyright: $\odot 2015$ Nipuni TKR, et al. This is an open-access article distributed under the terms of the Creative Commons Attribution License, which permits unrestricted use, distribution, and reproduction in any medium, provided the original author and source are credited.

\section{Short Communication}

Viral pathogens are more important microorganisms with respect to both medicinal and environmental aspects as it causes numerous diseases in humans, most of animals and plants. Therefore, wideranging studies on viral pathogens are conducted to identify viral species and to find diagnostic methods for viral diseases. Due to the shortcomings of traditional methods used to detect viral pathogens including cultured cell monolayers, antibody neutralization tests and molecular methods such as PCR [1]; a fast, cheap and emerging technology termed as 'metagenomic identification' has been introduced.

Metagenomics is a sequence-based analysis method which employs both improving sequencing technologies and computational capabilities [2]. It generally uses 'Sanger shotgun sequencing' method by which the DNA content of all viral pathogens in an environmental sample or a medical sample is sequenced in a random manner, thus the prerequisite of searching for a specific viral pathogen is not needed for the identification $[1,3]$. The genomic material used in shotgun sequencing for metagenomics is different from clonal culture genomics. In clonal culture genomics the raw genomic material comes from a single organism whereas, that of in metagenomics comes from a community of microbes [3].

The general procedure for the viral metagenome construction includes nine major steps:1) Sampling from habitat 2)The isolation of viral particles 3) Extraction of viral DNA and RNA 4) Reverse transcription of RNA to cDNA 5) Fragmentation of nucleic acids 6) Sequencing nucleic acid fragments7) Screening 8) Assembly and 9) Annotation [1,3]. The isolation is done using filtration, flocculation and density dependent centrifugation 1 to eliminate contaminating nonviral cells. Following fragmentation, the DNA fragments are cloned into plasmid vectors in order to obtain enough genome material for sequencing. Screening or computational filtering using bioinformatic methods are used to remove contaminating DNA after sequencing. Finally, short sequences are assembled into longer sequences and then into the whole genome using an assembly software followed by the annotation of assembled genome with reference genomes or genes.1,3Sequenced genomes can be further analyzed with sequences annotated as human pathogens.

Bioinformatic techniques are very important in viral metagenomics since it generates a large amount of sequence data including artifacts in metagenomic assembly such as chimeras and artificial repeats [1]. In identification of viral pathogens, bioinformatic annotation schemes like local alignments (BLAST, USEARCH) [1] are used with reference databases. Additional bioinformatic assessment (Gene calling, alignment with related proteins) [1] of the genome is needed in novel viral identification as it has no existing sequence data in databases. Except major steps, verification of viral identification by well-accepted methods like culturing or PCR is important. However, due to the lack of correlation between metagenomic annotation and PCR results1, further improvements of verification methods are required.

The metagenomic detection of viral pathogens can be applied in clinical diagnostics, public health monitoring and viral discovery [1]. Traditionally used culturing and diagnostic tests in clinical diagnosis of viral infections are incapable to identify rare and novel viruses; whereas, metagenomic identification overcomes these difficulties through the unambiguous and target-independent identification of viral pathogens as well as co-infections even in a single clinical sample. The outbreaks of viral pathogens also can be detected and responded by metagenomic identification which rapidly determines even viral subtypes of an outbreak and enables the correct therapeutic applications and prevention methods of epidemics. In viral discovery, metagenomic sequencing will help to understand viral pathogen diversity, to discover novel potentially pathogenic humanassociated viruses and to direct studies on novel pathogens. Environmental monitoring is an important aspect which employs target-independent metagenomic viral pathogen identification for research efforts, risk assessment and effective regulation of pathogens in complex environments such as sewage-polluted water and recreational beaches.

Limitations of metagenomic identification using shotgun sequencing include: providing of less sequence information, inability to confirm infectious nature of the virus and need of a pure isolate from a symptomatic patient with a novel virus to confirm causation [1]. Some of these can be overcome using methods like nextgeneration sequencing where the sequencing capacity is high [1].

In conclusion, there are significant advantages in viral metagenomics like target such as independent identification of pathogens, prior sequence information of a specific pathogen is not required, identification of multiple pathogens in a single sample and cost effectiveness. However, it needs further development in sequencing technologies, bioinformatics as well as molecular methods for the widespread adoption in various aspects.

\section{References}

1. Bibby K (2013) Metagenomic identification of viral pathogens. Trends Biotechnol 31: 275-279.

2. Handelsman J (2014) Metagenomics: application of genomics to uncultured microorganisms. Microbiol Mol Biol Rev 68: 669-685.

3. Wooley JC, Godzik A, Friedberg I (2010) A primer on metagenomics. In: Bourne PE (ed.) PLoS Comput Biol 6: e1000667. 